\title{
Magnetic domain replication in interacting bilayers with out-of-plane anisotropy: Application to Co/Pt multilayers
}

\author{
V. Baltz, ${ }^{1, *}$ A. Marty, ${ }^{2}$ B. Rodmacq, ${ }^{1}$ and B. Dieny ${ }^{1}$ \\ ${ }^{1}$ DRFMC/SPINTEC (URA CEA/CNRS 2512), CEA Grenoble, 17 Avenue des Martyrs, 38054 Grenoble Cedex 9, France \\ ${ }^{2}$ DRFMC/SP2M, CEA Grenoble, 17 Avenue des Martyrs, 38054 Grenoble Cedex 9, France
}

(Received 11 August 2006; revised manuscript received 13 November 2006; published 8 January 2007)

\begin{abstract}
The effects of domain replication resulting from interlayer magnetostatic coupling have been investigated in trilayers which consist of two Co/Pt multilayers with out-of-plane anisotropy separated by a thick Pt layer. For demagnetized films, the strong correlation between the domain configurations of the two multilayers gives rise to an evolution of the equilibrium domain size with the spacer layer thickness. A good correspondence is found between our numerical calculations and the experimental measurements. These results are an extension of Kaplan's theory of domain size in single ferromagnetic films with out-of-plane magnetization, to the case of interacting ferromagnetic films.
\end{abstract}

DOI: 10.1103/PhysRevB.75.014406

PACS number(s): 75.70.-i, 75.60.Ch, 75.75.+a

\section{INTRODUCTION}

A lot of experimental and theoretical work has been carried out so far in antiferromagnetically coupled multilayers or in "soft-hard" bilayers ${ }^{1-4}$ due to their technological potential for read-heads, magnetic random access memories, or multilevel recording applications. ${ }^{5-8}$

Possible interlayer magnetic coupling in such systems are susceptible to lead to misfunctionment of the devices. It has been shown for example in soft-hard bilayers with in-plane magnetization that the manipulation of the soft layer can influence the magnetic configuration of the hard layer. ${ }^{9-12}$

These couplings can have different origins: direct magnetic coupling through pinholes in the thin metallic or insulating spacer, $^{13}$ indirect exchange coupling through Ruderman-Kittel-Kasuya-Yosida (RKKY) interactions, ${ }^{14}$ orange peel (Néel) magnetostatic coupling in the presence of a correlated roughness at both spacer interfaces, ${ }^{15,16}$ and finally magnetostatic coupling through stray fields. ${ }^{16}$ In the latter case, although these interactions are mostly negligible in uniformly magnetized macroscopic samples, it is no longer the case when the magnetic layers are in a multidomain state, or when the lateral size of the sample is reduced.

So far, few studies on magnetostatic couplings in interacting bilayers have been carried out in systems with out-ofplane anisotropy. ${ }^{16-19}$ It is, however, noteworthy that, since in that case stray fields emanate from the domains themselves, different domain sizes or shapes lead to different stray field amplitudes.

In a single layer which exhibits perpendicular magnetic anisotropy, the evolution of the magnetic domain characteristic size is well described by Kaplan's analytical theory ${ }^{20}$ in the case where the film thickness is much smaller than the magnetic domain width and by Kittel's analytical theory in the case where the film thickness is much greater than the magnetic domain width. ${ }^{21}$ Extensions taking into account the " $\mu$ effect," 22 improved approximations ${ }^{23}$ and applications to $\mathrm{Co} / \mathrm{Pt}$ based systems ${ }^{24}$ have been developed. All these studies address the case of systems described as single layers.

Recently, more complex multilayer systems with out-ofplane anisotropy were studied such as exchange-biased ferromagnetic/antiferromagnetic bilayers, ${ }^{25}$ ferromagnetic/ nonmagnetic/ferromagnetic/antiferromagnetic multilayers ${ }^{16}$ or antiferromagnetically coupled ferromagnetic/ nonmagnetic/ferromagnetic films. ${ }^{14}$ In the latter system, domain formation at remanent states and magnetization reversal were linked to the field-induced phase transition in antiferromagnetic layers.

However, the interactions which may arise in systems of two ferromagnetic layers separated by a nonmagnetic interlayer in their lowest magnetic energy state have not been discussed at length so far, either experimentally nor theoretically. The purpose of this paper is to extend Kaplan's theory of magnetic domain size in single layers to the case of trilayers and to use this extension to quantitatively interpret an experimental investigation of the domain size in $\mathrm{Co} / \mathrm{Pt}$ based trilayers.

\section{SAMPLE PREPARATION}

Different series of samples were prepared by DC magnetron sputtering, with deposition rates of the order of $0.05 \mathrm{~nm} \mathrm{~s}^{-1}$ and $0.1 \mathrm{~nm} \mathrm{~s}^{-1}$ for cobalt and platinum, respectively. ${ }^{18}$ These series are of the generic form $\mathrm{Si} / \mathrm{SiO}_{2} / \mathrm{Pt} /[\mathrm{Co} / \mathrm{Pt}]_{n}, \mathrm{Si} / \mathrm{SiO}_{2} / \mathrm{Pt} /[\mathrm{Co} / \mathrm{Pt}]_{2} / \mathrm{Pt}(x) /[\mathrm{Co} / \mathrm{Pt}]_{4}$, and $\mathrm{Si} / \mathrm{SiO}_{2} / \mathrm{Pt} /[\mathrm{Co} / \mathrm{Pt}]_{4} / \mathrm{Pt}(x) /[\mathrm{Co} / \mathrm{Pt}]_{4}$. The number of repeats, $n$, ranges from 4 to 20 , the Pt spacer layer thickness, $x$, varies from 0 to $150 \mathrm{~nm}$, and $\mathrm{Co} / \mathrm{Pt}$ stands for a $[\mathrm{Co}(0.6 \mathrm{~nm}) / \mathrm{Pt}(1.8 \mathrm{~nm})]$ bilayer. The last two series will be abbreviated as $2 \times 4$ and $4 x 4$. Magnetic measurements were performed at room temperature using extraordinary Hall effect. ${ }^{26}$ Images of the magnetic domain configurations were obtained from magnetic force microscopy (MFM) in the demagnetized state.

Two Co layers separated by a $1.8 \mathrm{~nm}$ thick Pt film exhibit a strong ferromagnetic coupling $\left(J>3 \times 10^{-6} \mathrm{~J} \mathrm{~m}^{-3}\right)$. One can thus consider that the $\mathrm{Si} / \mathrm{SiO}_{2} / \mathrm{Pt} /[\mathrm{Co} / \mathrm{Pt}]_{n}$ samples are uniformly magnetized. On the other hand, two Co layers separated by a Pt film thicker than $4 \mathrm{~nm}$ exhibit a very small coupling $\left(J<3 \times 10^{-7} \mathrm{~J} \mathrm{~m}^{-3}\right)$ since the coupling energy due to pinholes, RKKY or orange peel mechanism decreases 


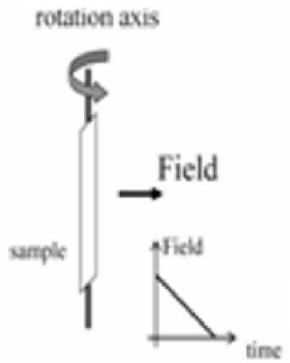

(a)

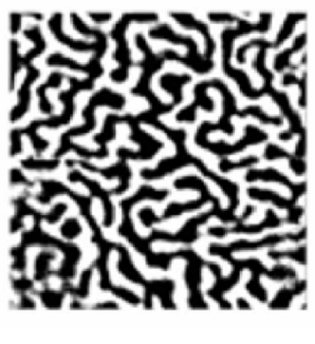

(b)

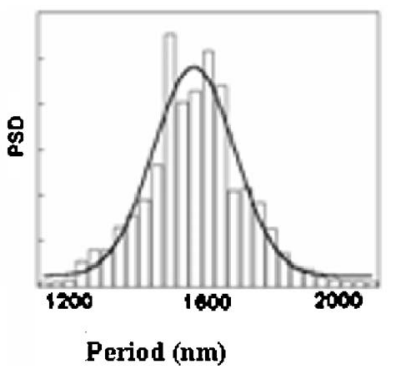

(c)
FIG. 1. (a) Schematic representation of the demagnetization procedure. (b) $20 \times 20 \mu \mathrm{m}^{2}$ MFM image for a $\mathrm{Si} / \mathrm{SiO}_{2} / \mathrm{Pt} /[\mathrm{Co} / \mathrm{Pt}]_{6}$ multilayer. (c) Fourier transform of the MFM image. The line is a Gaussian fit of the power spectral density (PSD). roughly exponentially with the nonmagnetic spacer thickness. ${ }^{16}$ In our case, in the $2 x_{x} 4$ and $4 x_{x} 4$ series (except when $x$ is smaller than $2.2 \mathrm{~nm}$ ), the spacing between the last Co sublayer of the bottom layer and the first Co sublayer of the top layer is larger than $4 \mathrm{~nm}(1.8 \mathrm{~nm}+2.2 \mathrm{~nm})$. Thus the two multilayers can be considered as two separated uniform magnetic layers with negligible interactions due to pinholes, RKKY or orange peel mechanism. This will allow us to probe only the magnetostatic interactions which will prevail in given configurations, such as for example the demagnetized configuration.

\section{EQUILIBRIUM DOMAIN SIZE IN Co/Pt ISOLATED MULTILAYERS}

Prior to the study of magnetostatically interacting multilayers in demagnetized configurations, we have checked the validity of the well known Kaplan model (evolution of the equilibrium domain size with the film thickness) for $\mathrm{Si} / \mathrm{SiO}_{2} / \mathrm{Pt} /[\mathrm{Co} / \mathrm{Pt}]_{\mathrm{n}}$ samples. $^{20,22,23}$ This study is a prerequisite and we will further refer to it in the following sections.

In order to reach a state as close as possible to the lowest energy one, and thus to have access to the equilibrium domain size, a demagnetization process was performed by rotating the samples $(180 \mathrm{rpm})$ in a slowly decreasing DC field (around $0.2 \mathrm{Oe} \mathrm{s}^{-1}$ ), as shown in Fig. 1(a). In this process, the rotation axis is in the plane of the sample and the field is applied perpendicular to this axis. The mean domain size is determined from MFM images. Figure 1(b) shows, as an example, the observed domain structure for a $\mathrm{Si} / \mathrm{SiO}_{2} / \mathrm{Pt} /[\mathrm{Co} / \mathrm{Pt}]_{6}$ sample. A two-dimensional (2D) Fourier transform of the MFM images allows us to obtain a histogram of the period distribution of the MFM images (i.e., twice the domain size) and their relative weight (PSD: power spectral density), as shown in Fig. 1(c). The distribution can be approximated by a Gaussian which mean value and standard deviation account for twice the mean domain size and error bars. The mean domain sizes that we measure are expected to be very close to the equilibrium ones, in the case of perfect stripe configurations. Indeed, the energy of stripe configurations increases strongly even with small changes in the domain period around its minimum. ${ }^{22}$

Figure 2 shows the evolution of the mean domain size, $d$, with the total film thickness, $t$, for a series of $\mathrm{Si} / \mathrm{SiO}_{2} / \mathrm{Pt} /[\mathrm{Co} / \mathrm{Pt}]_{n}$ multilayers, with $\mathrm{n}$ ranging from 4 to 20. It is noteworthy that the mean domain size decreases as the number of repeats (i.e., the total film thickness) increases.
The cost in domain wall energy is indeed compensated by the gain in demagnetizing energy as the film becomes thicker.

The full line is a fit to the Kaplan model that results from interplay between a gain in demagnetizing energy and a loss in domain wall energy. The predicted evolution of the equilibrium domain size with the film thickness is given by (Refs. 20, 22, and 23):

$$
\operatorname{Ln}\left(\frac{d}{t}\right)=\frac{\pi D_{0}}{2 t}+a
$$

for $\quad d / t>1.5, \quad$ with $\quad a=\operatorname{Ln}(\pi)-1+\mu\left(\frac{1}{2}-\operatorname{Ln}(2)\right), \quad$ and $\mu=1+\frac{2 \pi M_{s}^{2}}{K_{u}}$.

$M_{\mathrm{S}}$ and $K_{\mathrm{u}}$ correspond to the saturation magnetization and the uniaxial anisotropy constant, respectively, and are fixed parameters. $D_{0}$ stands for the dipolar length and is the only free parameter of the fit. The $\mu$ effect resulting from slight local misalignment of the magnetization with the anisotropy axis is taken into account. ${ }^{22,23}$

As discussed above, our multilayers can be considered as homogeneous thin films with a mean saturation magnetization equal to $340 \mathrm{emu} \mathrm{cm}^{-3}\left(M_{\mathrm{S}}=M_{\mathrm{Co}} t_{\mathrm{Co}} /\left(t_{\mathrm{Co}}+t_{\mathrm{Pt}}\right)\right.$ with $t_{\mathrm{Co}}$ and $t_{\mathrm{Pt}}$ the thicknesses of the $\mathrm{Co}$ and $\mathrm{Pt}$ sublayers). The uniaxial anisotropy has been deduced from vibrating sample magnetometry (VSM) measurements along the out-of-plane

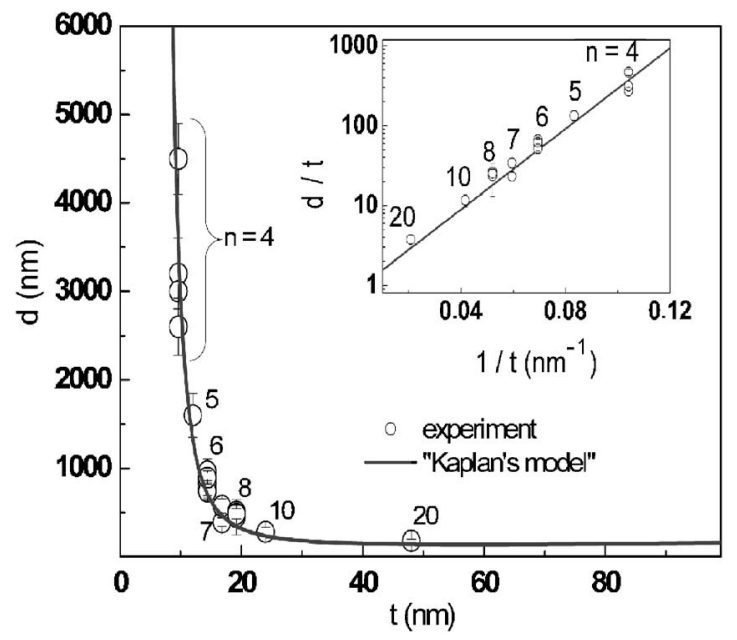

FIG. 2. Evolution of the mean equilibrium domain size, $d$, with the film thickness, $t$, for $\mathrm{Si} / \mathrm{SiO}_{2} / \mathrm{Pt} /[\mathrm{Co} / \mathrm{Pt}]_{\mathrm{n}}$ multilayers in the demagnetized state. The inset shows the evolution of $d / t$ as a function of $l / t$ in a semilogarithmic scale. 


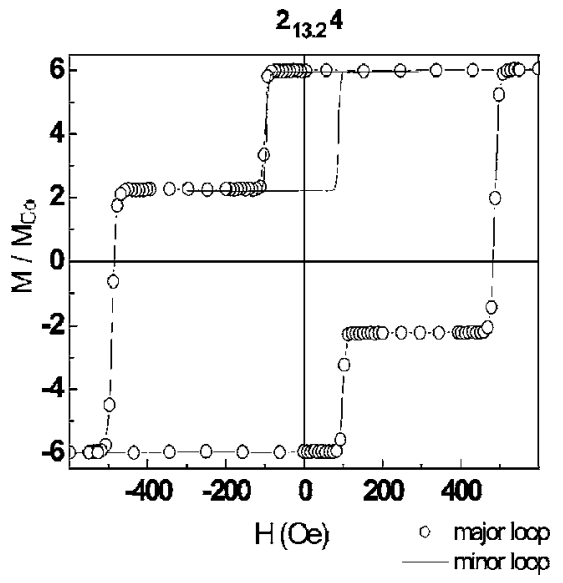

FIG. 3. Typical major and minor hysteresis loops measured by extraordinary Hall effect on a $\mathrm{Si} / \mathrm{SiO}_{2} / \mathrm{Pt} /[\mathrm{Co} / \mathrm{Pt}]_{2} / \mathrm{Pt}(13.2 \mathrm{~nm}) /[\mathrm{Co} / \mathrm{Pt}]_{4}$ film. The loops are normalized to the magnetization of a single Co layer.

easy axis and the in-plane hard axis of the samples. ${ }^{27}$ We find that, in the range of thicknesses studied, $K_{\mathrm{u}}$ is virtually independent of the sample thickness and is equal to $K_{\mathrm{u}}$ $=(1,6 \pm 0.2) \times 10^{6} \mathrm{erg} \mathrm{cm}^{-3}$. We thus obtain a quality factor $\left(K_{\mathrm{u}} / 2 \pi M_{\mathrm{s}}{ }^{2}\right)$ of 2.2 , in agreement with the hypothesis of a strong out-of-plane anisotropy required for the model. The total thickness of the films has been assumed to be: $t=\left(t_{\mathrm{Co}}\right.$ $\left.+t_{\mathrm{Pt}}\right) n$.

As can be seen in Fig. 2, a very good agreement between the experimental measurements and Kaplan's model is found and leads to $D_{0}=(39 \pm 2) \mathrm{nm}$. The knowledge of this parameter allows estimation of some of the physical parameters of the multilayers, such as the domain wall energy, $\sigma_{\mathrm{w}}$ $=2 \pi M_{\mathrm{S}}{ }^{2} D_{0}$, the exchange stiffness constant, $A$ $=\sigma_{\mathrm{w}}{ }^{2} /\left(16 \mathrm{~K}_{\mathrm{u}}\right)$, the Curie temperature, $T_{\mathrm{C}}=T_{\mathrm{C} \text {,bulk }} A / A_{\text {bulk }}$, with $A_{\text {bulk }} \sim 6.77 \times 10^{-7} \mathrm{erg} \mathrm{cm}^{-1}$ and $T_{\mathrm{C} \text {,bulk }} \sim 1390 \mathrm{~K}$ and the domain wall width, $l_{\mathrm{w}}=\pi\left(A / K_{\mathrm{u}}\right)^{1 / 2}$.

We thus calculate: $\sigma_{\mathrm{w}}=(2,8 \pm 0,2) \mathrm{erg} \mathrm{cm}^{-2}$, $A=(3.2 \pm 0.4) \times 10^{-7} \mathrm{erg} \mathrm{cm}^{-1}, \quad T_{\mathrm{C}}=(620 \pm 80) K, \quad l_{\mathrm{w}}$ $=(15 \pm 2) \mathrm{nm}$. Note that the hypothesis $l_{\mathrm{w}} \ll d$ of Kaplan's model is verified. The values that we obtain are in good agreement with the literature. ${ }^{24}$

\section{EQUILIBRIUM DOMAIN SIZE IN INTERACTING Co/Pt BASED TRILAYERS}

The aim of this part is the study of the effects resulting from magnetostatic interactions on the equilibrium domain size in a trilayer which consists of two demagnetized ferromagnets separated by a nonmagnetic layer.

\section{A. Experiments}

Figure 3 shows a typical hysteresis loop obtained by extraodinary Hall effect (sensitive to the perpendicular component of the magnetization) on a $\mathrm{Si} / \mathrm{SiO}_{2} / \mathrm{Pt} /[\mathrm{Co} / \mathrm{Pt}]_{2} / \mathrm{Pt}(13.2 \mathrm{~nm}) /[\mathrm{Co} / \mathrm{Pt}]_{4}:\left(2_{13.2} 4\right)$. As already mentioned, this notation means that the two nearest Co layers in each multilayer are separated by a $15 \mathrm{~nm}$ Pt layer.

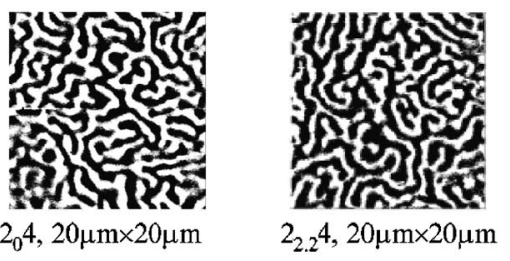

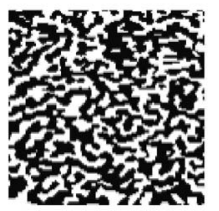

$2{ }_{13.2} 4,60 \mu \mathrm{m} \times 60 \mu \mathrm{m}$

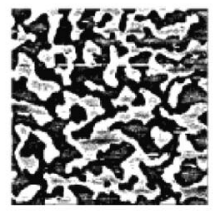

$228.24,60 \mu \mathrm{m} \times 60 \mu \mathrm{m}$

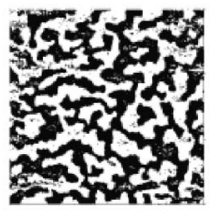

$2_{98.2} 4,60 \mu \mathrm{m} \times 60 \mu \mathrm{m}$
FIG. 4. MFM images for $\mathrm{Si} / \mathrm{SiO}_{2} / \mathrm{Pt} /[\mathrm{Co} / \mathrm{Pt}]_{2} / \mathrm{Pt}(x) /[\mathrm{Co} / \mathrm{Pt}]_{4}$ films $\left(2{ }_{\mathrm{x}} 4\right)$ for different Pt spacer thicknesses.

The two ferromagnetic layers display square loops and exhibit different coercitivities so that successive switching of the magnetization in the layers is observed. ${ }^{18}$ This is due to both their different thicknesses (two repeats for the bottom layer and four repeats for the top one) and the different thicknesses of the buffer on which they grow (1.8 nm of Pt for the bottom layer, and $15 \mathrm{~nm}$ for the top layer). ${ }^{24}$ Note that the very small shift of the minor loop $(\sim 5 \mathrm{Oe})$ accounts for negligible interlayer interactions due to possible pinholes, RKKY or orange peel coupling, as justified above. ${ }^{18}$

A series of typical MFM images is shown in Fig. 4, for $\mathrm{Si} / \mathrm{SiO}_{2} / \mathrm{Pt} /[\mathrm{Co} / \mathrm{Pt}]_{2} / \mathrm{Pt}(x) /[\mathrm{Co} / \mathrm{Pt}]_{4}$ films in the demagnetized configuration, with varying values of the $\mathrm{Pt}$ spacer thickness, $x$. The samples have been demagnetized using the same procedure as that described in the previous section.

For thin Pt spacer thicknesses, the MFM allows us to probe the stray field resulting from the bottom layer combined with that from the top layer. The images display dark and bright contrasts. The absence of distinguishable intermediate gray contrast in the image, and thus of intermediate "up-down" and "down-up" states, results from domain replication ascribed to strong interlayer magnetostatic interactions. ${ }^{18}$ Note that the absence of such intermediate contrast has been confirmed by polar MOKE microscopy measurements (not shown) which also proved to be efficient in detecting such contrast. ${ }^{19}$ The dark and bright contrasts observed in Fig. 4 thus respectively correspond to configurations where the bottom and top layers are saturated up-up or down-down.

For thick Pt spacer thicknesses, it is likely that the stray field from the bottom layer becomes too weak and that only the stray field resulting from the top layer is probed. The observed contrast thus corresponds to the up or down configurations of the top layer. However, in the case of replicated domains, these contrasts from the top layer also well describe the bottom replicated layer.

From Fig. 4, one notes that the system displays smaller domains when the Pt spacer thickness is reduced. This is in qualitative agreement with Kaplan's model previously described in the case of a single noninteracting layer (Fig. 2). Indeed, in the case of two interacting layers, the thinner the spacer, the larger the interlayer magnetostatic interactions, 


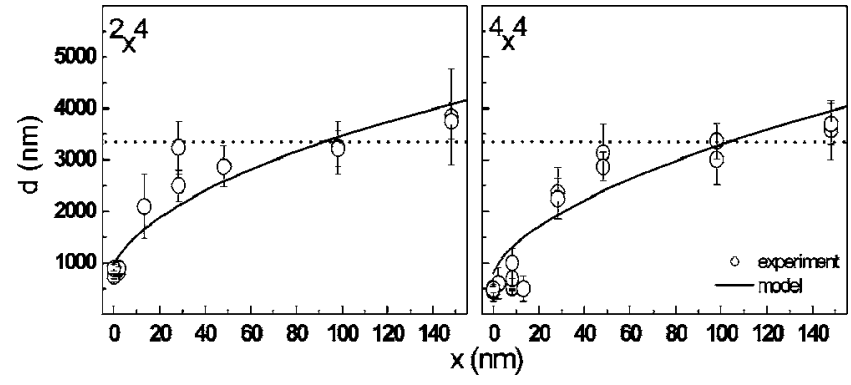

FIG. 5. Evolution of the mean equilibrium domain size, $d$, with the $\mathrm{Pt}$ spacer thickness, $x$, for $\mathrm{Si} / \mathrm{SiO}_{2} / \mathrm{Pt} /[\mathrm{Co} / \mathrm{Pt}]_{\mathrm{n}} / \mathrm{Pt}(x) /[\mathrm{Co} / \mathrm{Pt}]_{4}$ multilayers in the demagnetized state, with $n=2$ and 4 . The dashed lines indicate the order of magnitude of the domain size for a single $\mathrm{Si} / \mathrm{SiO}_{2} / \mathrm{Pt} /[\mathrm{Co} / \mathrm{Pt}]_{4}$ multilayer. The plain lines result from fits using the model described in the text.

and the thicker the "equivalent" total thickness of the film.

Figure 5 shows the evolution of the mean domain size, $d$, with the $\mathrm{Pt}$ spacer thickness, $x$, for $\mathrm{Si} / \mathrm{SiO}_{2} / \mathrm{Pt} /[\mathrm{Co} / \mathrm{Pt}]_{n} / \mathrm{Pt}[x] /[\mathrm{Co} / \mathrm{Pt}]_{4}$ systems, with $n=2$ and $n=4$. The mean domain size has been deduced from the Fourier transform of the MFM images, as previously detailed. Before trying to model such evolutions, we will describe qualitatively the behavior of the layers domain configurations, as the spacer thickness is varied.

For the thicker Pt spacer thickness probed $(148.2 \mathrm{~nm})$, the mean domain size is of the same order of magnitude as that of a single multilayer with four repeats (mean value indicated by the dashed lines in Fig. 5, and deduced from the results shown in Fig. 2). For such a spacer thickness, it is likely that we reach the limiting case in which the bottom and top layers no longer interact. The stray field from each layer probably close within each layer, as schematically displayed in Fig. 6(a). The mean domain sizes associated to each individual layer are thus observed, i.e., around $3.5 \mu \mathrm{m}$ for the top layer with four repeats, and $400 \mu \mathrm{m}$ for the bottom layer with two repeats, this latter value being extrapolated using Kaplan's model. However, it is likely that for such very small thicknesses, the magnetic configuration rather displays bubbles than stripe domains.

For the thinner spacer regime, the mean domain size decreases as the spacer thickness is reduced (Fig. 5). As the domain sizes of the bottom and top layers become much larger than the spacer thickness, the magnetic configuration of the layers are identical and match, resulting from the strong interlayer magnetostatic interactions, as schematically displayed in Fig. 6(b), and previously evidenced. ${ }^{18}$ Indeed, it was shown in Ref. 18, that it is even possible to take advan- tage of domain replication in order to probe the microscopic domain configuration of the hard layer through macroscopic hysteresis minor loop measurements on the soft layer.

Due to domain replication, the mean domain size that we obtain is smaller than the domain sizes of the noninteracting layers. The stronger the interactions, the more the $2{ }_{x} 4$ and $4_{x} 4$ samples behave like unique multilayers with six or eight repeats. According to Fig. 2 (Kaplan model), the domain sizes of these unique multilayers with 6 or 8 layers are smaller than that of unique multilayers with 2 or 4 repeats, and one measures mean domain sizes around $800 \mathrm{~nm}$ and $500 \mathrm{~nm}$, as shown in Fig. 5.

\section{B. Model}

We will now present a model which describes the evolution of the mean domain size with the spacer layer thickness for two ferromagnetic layers spaced by a nonmagnetic layer, in the demagnetized state. We only consider the case of replicated domains, as shown in Fig. 6(b), and inferred from the experimental dependence of the mean domain size with the spacer layer thickness.

We consider two ferromagnetic layers of thicknesses $t_{\mathrm{n}}$ and $t_{\mathrm{m}}$, with the same out-of-plane uniaxial magnetic anisotropy per unit volume $K_{\mathrm{u}}$ and the same saturation magnetization $M_{\mathrm{s}}$, separated by a nonmagnetic layer of thickness $x$. We are interested here in the lowest energy configuration of the trilayer. These layers are both thin enough to consider that the lowest energy state is made of stripe domains with equal numbers of up and down domains within each layer. We consider that couplings through pinholes, RKKY or orange peel mechanism are negligible. We study the equilibrium domain size in zero field so that no Zeeman energy is included in the total energy. The $180^{\circ}$ Bloch walls [characterized by an energy per unit area $\left.\sigma_{\mathrm{w}}(u)\right]$ are assumed to be perpendicular to the sample surface. Moreover, we consider the case of a strong uniaxial anisotropy $K_{\mathrm{u}}>2 \pi \mathrm{M}_{\mathrm{s}}{ }^{2}$ so that the domain wall width is much smaller than the domain size. As a consequence, the gradient of anisotropy and the exchange energy are localized in the domain walls and are independent of the magnetic domain size.

The total energy per unit surface can then be written

$$
E_{t}=\sigma_{w}\left(\beta_{n}\right)+\sigma_{w}\left(\beta_{m}\right)+E_{d}\left(\beta_{n}, \beta_{m}, \beta_{x}\right),
$$

with $\beta_{i}=\frac{t_{i}}{d}$ and $\beta_{x}=\frac{x}{d}$.

The first two terms of Eq. (2) correspond to the wall energy per unit surface of the two layers with $\sigma_{\mathrm{w}}=4\left(A K_{\mathrm{u}}\right)^{1 / 2}$ $=2 \pi M_{\mathrm{S}}{ }^{2} D_{0}$, where $D_{0}$ is the dipolar length. We consider that $D_{0}$ is identical for both layers. The last term of Eq. (2)
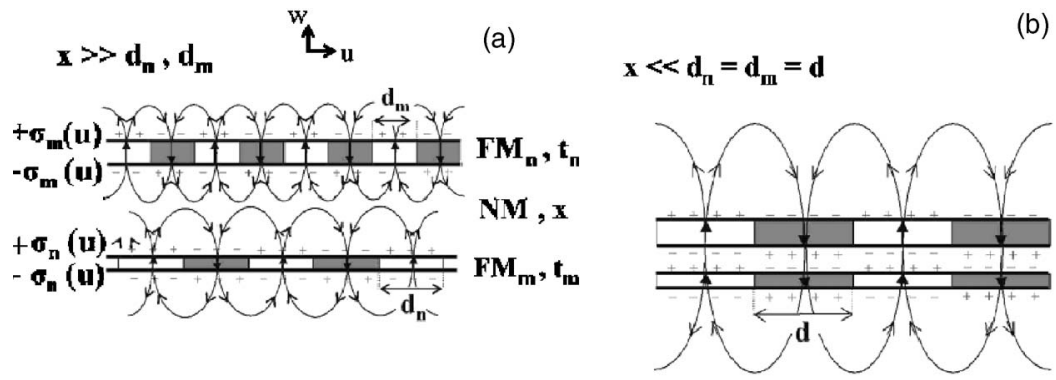

FIG. 6. Schematic 2D representation of a ferromagnetic/nonmagnetic/ferromagnetic trilayer in its lowest energy configuration for two limiting cases: (a) when $x \ll d_{\mathrm{n}}=d_{\mathrm{m}}=d$ and (b) when $x \gg d_{\mathrm{n}}, d_{\mathrm{m}} . \sigma(u)$ is the interfacial surface energy distribution. 
corresponds to the magnetostatic energy per unit surface of the system. This energy can be extended from the single layer expression by taking into account the interactions between all the magnetically charged surfaces, i.e., the cross and self-interactions between the four charged surfaces, knowing that two charged surfaces per magnetic layer are considered. The sum of the self energies of all the four charged surfaces cancel out each other and thus the magnetostatic energy of the system can be written

$$
\begin{aligned}
E_{d}\left(\beta_{n}, \beta_{m}, \beta_{x}\right)= & e_{d}\left(\beta_{n}\right) t_{n}+e_{d}\left(\beta_{m}\right) t_{m} \\
& +e_{d}\left(\beta_{n}+\beta_{m}+\beta_{x}\right)\left(t_{n}+t_{m}+x\right) \\
& +e_{d}\left(\beta_{x}\right) x-e_{d}\left(\beta_{n}+\beta_{x}\right)\left(t_{n}+x\right) \\
& -e_{d}\left(\beta_{m}+\beta_{x}\right)\left(t_{m}+x\right),
\end{aligned}
$$

where $e_{\mathrm{d}}\left(\beta_{\mathrm{i}}\right)$ is the demagnetizing energy per unit volume of a single layer with periodic stripe domains. $e_{\mathrm{d}}\left(\beta_{\mathrm{n}}\right), e_{\mathrm{d}}\left(\beta_{\mathrm{m}}\right)$, $e_{\mathrm{d}}\left(\beta_{\mathrm{x}}\right), e_{\mathrm{d}}\left(\beta_{\mathrm{n}}+\beta_{\mathrm{m}}+\beta_{\mathrm{x}}\right), e_{\mathrm{d}}\left(\beta_{\mathrm{n}}+\beta_{\mathrm{x}}\right)$, and $e_{\mathrm{d}}\left(\beta_{\mathrm{m}}+\beta_{\mathrm{x}}\right)$ are the energies resulting from the six crossed interactions between the four charged surfaces. They would be equivalent to demagnetizing energies per unit volume of six virtual single layers with periodic stripe domains, and separated by $t_{\mathrm{n}}, t_{\mathrm{m}}$, $x, t_{\mathrm{n}}+t_{\mathrm{m}}+x, t_{\mathrm{n}}+x$, and $t_{\mathrm{m}}+x$, respectively. These energies can be deduced from Eq. (A.4) of Ref. 23 with the thin film limit condition $\beta<0.6$ and is given by:

$$
e_{d}(\beta)=2 \pi M_{s}^{2}\left[1+\frac{2}{\pi} \beta(\operatorname{Ln}(\beta)+a-1)\right],
$$

with $a=\operatorname{Ln}(\pi)-1+\mu\left(\frac{1}{2}-\operatorname{Ln}(2)\right)$, and $\mu=1+\frac{2 \pi M_{s}^{2}}{K_{u}}$.

The variation of the equilibrium domain size versus the spacer thickness is deduced by first injecting Eqs. (3) and (4) in Eq. (2) and then from the minimization of the resulting expression of the total energy with respect to $d$. This energy minimization yields the following relation:

$$
\operatorname{Ln}\left(\frac{d}{t_{n}+t_{m}}\right)=\frac{\pi D_{0}}{2\left(t_{n}+t_{m}\right)}+a+c(x),
$$

with $c(x)=\mathbf{s}\left(t_{n}\right)+\mathbf{s}\left(t_{m}\right)+\mathbf{s}\left(t_{n}+t_{m}+x\right)+\mathbf{s}(x)-\mathbf{s}\left(t_{n}+x\right)-\mathbf{s}\left(t_{m}+x\right)$, and $s\left(t_{i}\right)=\left(\frac{t_{i}}{t_{n}+t_{m}}\right)^{2} \operatorname{Ln}\left(\frac{t_{i}}{t_{n}+t_{m}}\right)$.

Note that when $x$ goes to zero, which corresponds to the case of a single layer with a total thickness equal to $t_{\mathrm{n}}+t_{\mathrm{m}}, c$ goes to zero and Eq. (5) becomes equivalent to the relation derived by Kaplan [Eq. (1)]. ${ }^{20,22,23}$

The theoretical model is now compared to the experimental results. The fixed parameters of the model are the layers thicknesses $\left(t_{\mathrm{n}}=4.8 \mathrm{~nm}\right.$ for the $22_{\mathrm{x}} 4$ samples and $t_{\mathrm{n}}=9.6 \mathrm{~nm}$ for the $44_{\mathrm{x}} 4$ samples, $\left.t_{\mathrm{m}}=9.6 \mathrm{~nm}\right)$, the saturation magnetization $\left(M_{\mathrm{S}}=340 \mathrm{emu} \mathrm{cm}^{-3}\right)$, and the anisotropy constant $\left(K_{\mathrm{u}}\right.$ $\left.=1,6 \times 10^{6} \mathrm{erg} \mathrm{cm}^{-3}\right)$. There is only one adjustable parameter, the dipolar length $D_{0}$.
The variation of the magnetic domain size with the spacer thickness is plotted in Fig. 5 for the $\mathrm{Si} / \mathrm{SiO}_{2} / \mathrm{Pt} /(\mathrm{Co} / \mathrm{Pt})_{\mathrm{n}} / \mathrm{Pt}_{\mathrm{x}} /(\mathrm{Co} / \mathrm{Pt})_{4}$ systems, with $n=2$ and $n=4$, together with the results of the fits that allow getting the relevant trend, i.e., an increase of the domain size as the spacer thickness increases.

From these fits, we obtain values of $D_{0}=40 \mathrm{~nm}$ and $49 \mathrm{~nm}$ for $n=2$ and $n=4$, respectively. These values agree with the order of magnitude previously deduced from Kaplan's model $\left(D_{0}=39 \mathrm{~nm}\right)$ in the case of noninteracting multilayers with varying numbers of repeats. Such a dipolar length previously allowed us to determine realistic values of exchange stiffness constant, Curie temperature, and domain wall width.

We can then check the validity of the model: (i) we calculate $\beta_{\max }=\left[\left(t_{\mathrm{n}}+t_{\mathrm{m}}+x\right) / d\right]_{\max } \sim 0.03 \ll 0.6$ which is in agreement with the assumption of Eq. (3); (ii) we observe $d \gg x$ in the whole range of spacer thickness investigated; (iii) from the dipolar length $D_{0}$, we obtain the following domain wall width: $l_{\mathrm{w}}=15 \mathrm{~nm}$ and $l_{\mathrm{w}}=20 \mathrm{~nm}$ for $n=2$ and $n=4$, values which are indeed very small compared to the mean magnetic domain sizes.

It is noteworthy that this simplified model seems to indicate that domain replication occurs in the whole range of thicknesses studied, contrary to our qualitative expectations. Note however, that this model is no longer valid in the limiting case of noninteracting layers.

\section{CONCLUSION}

To conclude, we proposed an extension of Kaplan's theory of the evolution of the magnetic domain size in ferromagnetic films with perpendicular magnetization for multilayered systems, by taking into account the interlayer coupling interactions. We confronted the model to experimental results on Co/Pt based demagnetized continuous films and we have shown that strong magnetic stray fields resulting from the finite size of the domains in systems can lead to domain replication in trilayers which consist of two ferromagnets spaced by a nonmagnetic layer. Such systems have notably been suggested as multilevel magnetic media in order to possibly increase the data storage densities. ${ }^{7}$ The present study suggests that thick interlayer spacer or interlayer shields will be needed for the implementation of such devices. Based upon domain replication, it has also recently been envisaged to probe the microscopic magnetic domain configuration of a hard layer through macroscopic hysteresis minor loops on an interacting softer layer. ${ }^{18}$ This work may, for example, also be useful for the quantitative interpretation of such data.

\section{ACKNOWLEDGMENT}

The authors acknowledge A. T. Hindmarch from the EC Stoner Laboratory of the University of Leeds for the critical reading of the manuscript. 
*Present address: School of Physics \& Astronomy, University of Leeds, Leeds LS2 9JT, United Kingdom. Electronic address: v.a.baltz@leeds.ac.uk

${ }^{1}$ M. N. Baibich, J. M. Broto, A. Fert, F. Nguyen Van Dau, F. Petroff, P. Etienne, G. Creuzet, A. Friederich, and J. Chazelas, Phys. Rev. Lett. 61, 2472 (1988).

${ }^{2}$ G. Binasch, P. Grünberg, F. Saurenbach, and W. Zinn, Phys. Rev. B 39, 4828 (1989).

${ }^{3}$ S. S. P. Parkin, N. More, and K. P. Roche, Phys. Rev. Lett. 64, 2304 (1990).

${ }^{4}$ B. Dieny, V. S. Speriosu, S. S. P. Parkin, B. A. Gurney, D. R. Wilhoit, and D. Mauri, Phys. Rev. B 43, 1297 (1991).

${ }^{5}$ B. Dieny, in Magnetoelectronics (Elsevier Academic Press, Amsterdam, 2004), p. 67.

${ }^{6}$ M. G. Samant and S. S. P. Parkin, Vacuum 74, 705 (2004).

${ }^{7}$ V. Baltz, S. Landis, B. Rodmacq, and B. Dieny, J. Magn. Magn. Mater. 290-291, 1286 (2005).

${ }^{8}$ M. Albrecht, G. Hu, A. Moser, O. Hellwig, and B. D. Terris, J. Appl. Phys. 97, 103910 (2005).

${ }^{9}$ L. Thomas, M. G. Samant, and S. S. P. Parkin, Phys. Rev. Lett. 84, 1816 (2000).

${ }^{10}$ C. Cowache, B. Dieny, S. Auffret, M. Cartier, R. H. Taylor, R. O'Barr, and S. Y. Yamamoto, IEEE Trans. Magn. 34, 843 (1998).

${ }^{11}$ W. S. Lew, S. P. Li, L. Lopez-Diaz, D. C. Hatton, and J. A. C. Bland, Phys. Rev. Lett. 90, 217201 (2003).

${ }^{12}$ W. Kuch, L. I. Chelaru, K. Fukumoto, F. Porrati, F. Offi, M. Kotsugi, and J. Kirschner, Phys. Rev. B 67, 214403 (2003).
${ }^{13}$ J. Wang, Y. Liu, P. P. Freitas, E. Snoeck, and J. L. Martins, J. Appl. Phys. 93, 8367 (2003).

${ }^{14}$ O. Hellwig, T. L. Kirk, J. B. Kortright, A. Berger, and E. E. Fullerton, Nat. Mater. 2, 112 (2003).

${ }^{15}$ L. Néel, C. R. Hebd. Seances Acad. Sci. 255, 1676 (1962).

${ }^{16}$ J. Moritz, F. Garcia, J. C. Toussaint, B. Dieny, and J. P. Nozières, Europhys. Lett. 65, 123 (2004).

${ }^{17}$ H. W. Fuller and D. L. Sullivan, J. Appl. Phys. 33, 1063 (1962).

${ }^{18}$ B. Rodmacq, V. Baltz, and B. Dieny, Phys. Rev. B 73, 092405 (2006).

${ }^{19}$ S. Wiebel, J.-P. Jamet, N. Vernier, A. Mougin, J. Ferré, V. Baltz, B. Rodmacq, and B. Dieny, Appl. Phys. Lett. 86, 142502 (2005); J. Appl. Phys. 100, 043912 (2006).

${ }^{20}$ B. Kaplan and G. A. Gehring, J. Magn. Magn. Mater. 128, 111 (1993).

${ }^{21}$ C. Kittel, Phys. Rev. 70, 965 (1946).

${ }^{22}$ C. Kooy and U. Enz, Philips Res. Rep. 15, 7 (1960).

${ }^{23}$ V. Gehanno, Y. Samson, A. Marty, B. Gilles, and A. Chamberod, J. Magn. Magn. Mater. 172, 26 (1997).

${ }^{24}$ S. Landis, B. Rodmacq, and B. Dieny, Phys. Rev. B 62, 12271 (2000); S. Landis, Ph.D. thesis, Université Joseph Fourier de Grenoble, 2001.

${ }^{25}$ J. Nogués and I. K. Schuller, J. Magn. Magn. Mater. 192, 203 (1999).

${ }^{26}$ C. L. Canedy, X. W. Li, and G. Xiao, J. Appl. Phys. 81, 5367 (1997).

${ }^{27}$ S. Chikazumi, Physics of Magnetism (Wiley and Sons, New York, 1964). 